See discussions, stats, and author profiles for this publication at: https://www.researchgate.net/publication/330442520

\title{
Least-weight composite plates with unconventional stacking sequences: Design, analysis and experiments
}

Article in Journal of Composite Materials · January 2019 DOI: $10.1177 / 0021998318824783$

\section{CITATIONS}

4 authors:

Marco Montemurro

Ecole Nationale Supérieure d'Arts et Métiers

87 PUBLICATIONS 528 CITATIONS

SEE PROFILE

Jalal El Yagoubi

Institut de Mécanique et d'Ingénierie de Bordeaux

22 PUBLICATIONS 111 CITATIONS

SEE PROFILE
READS

207

Michele lacopo Izzi

Ecole Nationale Supérieure d'Arts et Métiers

6 PUBLICATIONS 2 CITATIONS

SEE PROFILE

Daniele Fanteria

Università di Pisa

33 PUBLICATIONS 135 CITATIONS

SEE PROFILE

Some of the authors of this publication are also working on these related projects:

VulComp2 [ANR "MATETPRO" Project] View project

Analysis and optimum Design of Additive Manufactured composite strUctures for Space applications (ADAMUS) View project 


\title{
Least-weight composite plates with unconventional stacking sequences: design, analysis and experiments
}

\author{
Marco Montemurro ${ }^{\mathrm{a}, *}$, Michele Iacopo Izzi ${ }^{\mathrm{a}}$, Jalal El-Yagoubi ${ }^{\mathrm{b}}$, Daniele Fanteria ${ }^{\mathrm{c}}$ \\ ${ }^{a}$ Arts et Métiers Paris Tech, I2M CNRS UMR 5295, F-33400 Talence, France \\ ${ }^{b}$ Université de Bordeaux, I2M CNRS UMR 5295, F-33400 Talence, France \\ ${ }^{c}$ University of Pisa, Department of Civil and Industrial Engineering - Aerospace division, \\ Via G. Caruso 8, 56122 Pisa, Italy
}

\begin{abstract}
This study deals with the problem of the least-weight design of a composite multilayer plate subject to constraints of different nature (mechanical, geometrical and technological requirements). To face this problem, a multi-scale two-level (MS2L) design methodology is proposed. This approach aims at optimising simultaneously both geometrical and mechanical parameters of the laminate at each characteristic scale (mesoscopic and macroscopic ones). In this background, at the first level (macroscopic scale) the goal is to find the optimum value of geometrical and mechanical design variables minimising the structure mass and satisfying the set of imposed constraints (on first buckling load, membrane stiffness and feasibility constraints). The second-level problem (mesoscopic scale) aims at finding at least one stacking sequence meeting the geometrical and material parameters provided by the first-level problem. The MS2L optimisation approach is based on the polar formalism to describe the macroscopic behaviour of the composite (in the framework of the equivalent single layer theories) and on a special genetic algorithm to perform optimisation calculations. The optimum solutions provided by the MS2L optimisation strategy are characterised by a weight saving of about $10 \%$ with enhanced mechanical properties when compared to conventional symmetric balanced stacks. The effectiveness of the optimum solutions is also proven through an experimental campaign of buckling tests. The experimental results are in excellent agreement with those foreseen by the numerical simulations.
\end{abstract}

Keywords: Composites, Finite Element Method, Buckling, Multi-scale Optimisation, Lightweight structures, Genetic Algorithm.

This is a pre-print of an article published in Journal of Composite Materials. The final authenticated version is available online at: https://doi.org/10.1177/0021998318824783

\footnotetext{
*Corresponding author. Tel.: +33 556845 422, Fax.: +33 540006964 .

Email address: marco.montemurro@ensam.eu; marco.montemurro@u-bordeaux.fr (Marco Montemurro)
} 


\section{Introduction}

The constant demand of lightweight structures with enhanced mechanical performances has led to an increasing use of composite materials in the last few decades. Fibre-reinforced composites present specific stiffness and strength properties that make them really appealing when compared to metallic alloys. The behaviour of these materials gives the designer the opportunity to tailor the material properties according to the design needs. A lot of research has been carried out in looking for the best strategy to optimise multilayer composite plates in order to either minimise the mass (without loosing performances with respect to a given reference solution) or improve mechanical performances (without increasing the mass with respect to a reference configuration). Nonetheless, the problem is still open. The design of a composite structure is a quite difficult problem that can be considered as a multi-scale optimisation problem. The complexity of the design process is due to two intrinsic properties of composite materials, i.e. heterogeneity and anisotropy. Heterogeneity gets involved mainly at the microscopic scale (i.e. that of constitutive phases), whilst anisotropy intervenes at both mesoscopic scale (that of the lamina) and macroscopic one (that of the laminate). To deal with heterogeneity a common strategy is to make use of homogenization techniques [1-3].

The main consequence of anisotropy is the introduction of some phenomena and issues that do not exist in metals (e.g. extension-bending coupling, delamination, residual stresses, free-edge stresses, different failure mechanisms, etc.). A further complication is that the design process must deal with a significant amount of design variables at different scales. In fact, up to now, no general methods exist for the optimum design of a composite structure.

Adali et al. [4] investigated the post-buckling stiffness maximisation problem of simply supported biaxially loaded laminated plates using five pre-set symmetrical angle-ply stacking sequences consisting of eight plies. In this way the number of design variables reduces to one (for each configuration) and the optimisation was carried out by means of the Golden Section method. Later [5] they focused on the problem of maximising the first buckling load of a bi-material multilayer plate. In each considered case the stacking sequence is balanced and symmetric and the orientations are limited to the canonical set $\left\{0^{\circ}, \pm 45^{\circ}, 90^{\circ}\right\}$. Finally in $[6,7]$ both approaches have been used in the framework of multi-objective optimisation problems.

Haftka and Walsh [8] used integer programming for the optimum design of symmetric balanced stacks wherein the orientations are constrained to get the values in the canonical set. These problems were solved by considering additional requirements on the percentage rule and overall in-plane stiffness value. Le Riche and Hafka [9] made use of a genetic algorithm (GA) to perform the solution search for the problem of maximising the first buckling load of a multilayer plate with a given number of plies. Also in this work, symmetric balanced stacks with orientation angles getting values in the canonical set were considered. Furthermore, an ad-hoc genetic operator, i.e. the permutation operator, was proposed to increase the efficiency of the calculations.

Still in the context of the first buckling load maximisation problem, Aymerich and Serra [10] utilised the Ant Colony Optimisation method (considering balanced symmetric stacking sequences in the usual domain of orientations) which was characterised by a better efficiency, in terms of computational effort, when compared to GA-based strategies. Irisarri et al. [11] performed a multi-objective optimisation process of laminated plates using a Pareto-based evolutionary algorithm. In this interesting work symmetric balanced stacks are employed and the orientation of each couple of plies comes from a set bigger than the canonical one, i.e. $\left(0_{2}^{\circ}, \pm 15^{\circ}, \pm 30^{\circ}, \ldots, 90_{2}^{\circ}\right)$. Furthermore, a set of design guidelines [12] are 
integrated in the optimisation process, though the flexural behaviour of the laminate was only approximated.

The previous works aimed at solving the design problem by directly optimising the value of the layers orientation angles without introducing a multi-scale design/optimisation approach. In each study, the nature of the stack is set a priori and the orientation angles are limited to get values in a predefined set. Moreover, further design (empirical) rules [12] were integrated as optimisation constraints. All these aspects contribute to strongly shrink the design domain leading the algorithm to find only suboptimal solutions. All the previous works are based on an analytical formulation of the optimisation problem. Conversely, for real-world engineering problems the mechanical response of the structure is typically evaluated by means of a suitable finite element (FE) model.

In this scenario, multi-scale optimisation strategies of composite structures which aim at formulating and solving the design/optimisation problem at each pertinent scale (without introducing simplifying hypotheses on both the nature of the stacking sequence and on the value of plies orientation) find strong motivations. When dealing with the multi-scale optimisation problem of a composite, at the macroscopic scale, the behaviour is described in terms of the laminate stiffness tensors components, regardless of the nature of the stack. When the laminate behaviour is expressed in the framework of the classic laminate theory (CLT) the Cartesian components of membrane, bending and membrane/bending coupling stiffness tensors constitute the unknowns of the problem. However, since Cartesian components are frame dependent, an alternative representation of such tensors is often used. The most common approach makes use of the well-known lamination parameters (LPs) coupled with the parameters of Tsai and Pagano [13]. These parameters [14, 15] unquestionably provide a compact representation of the stiffness tensors of composite laminates in the framework of the CLT, although they are not all tensor invariants [13].

Diaconu et al. [16] presented a general framework for determining the feasible region in LPs space for general composite laminate design. Their method does not give an explicit relationship between LPs, but only a "numerical definition" of the boundary of the feasible domain. Later they presented a work on the layup optimisation of thick laminates for maximising the first natural frequency [17]. In this context, a multi-step optimisation approach including numerical verification on the feasibility of the optimum solutions is proposed. Liu et al. [18] considered the problem of the maximisation of the first buckling load of a multilayer plate in the flexural LPs space. The optimum solutions are only given in terms of flexural LPs characterising the equivalent homogeneous anisotropic plate.

Bloomfield et al. [19] presented a two-step optimisation strategy for symmetric laminates made of a predefined set of possible ply orientations. The strategy is applied to the problem of mass minimisation of a simply supported multilayer plate under different loading conditions. The results highlight the interest in widening the standard canonical set by adding $\pm 30^{\circ}$ and $\pm 60^{\circ}$ values. Liu et al. [20] presented a two-step optimisation strategy for maximising the stiffness of laminates subject to a given set of optimisation constraints. During the first step the optimisation problem is solved in the LPs space wherein the feasible region has been approximated by the one that can be obtained by considering only six different orientation angles. During the second step, a suitable stack is retrieved by solving a least-square problem in which the orientation angles of the laminae are the design variables.

As it can be inferred from the previous works, the multi-scale optimisation approach based on LPs presents two main weaknesses: LPs are not tensor invariants, while not all Tsai and Pagano parameters are invariants; both LPs and Tsai and Pagano parameters have not an immediate physical meaning related to the elastic symmetries of the stiffness tensor. 
Moreover, although the previous studies made use of the multi-scale optimisation strategy based on LPs, an unnecessary restriction still remains when looking for the optimum stack: in the formulation of the laminate lay-up design problem simplifying hypotheses on the nature of the stacking sequence are systematically used. These assumptions are used, on the one hand, to obtain a short-cut to a possible solution (i.e. to obtain some desired mechanical properties). On the other hand, the aim of these rules is to prevent the laminate from some undesired phenomena, though this is never clearly and rigorously stated nor proved. Unfortunately, the use of these simple rules shrinks the design space and drives the optimisation algorithm towards suboptimal solutions.

Two examples are the use of symmetric stacking sequences (a sufficient but not necessary condition for membrane-bending uncoupling) and the use of balanced stacks to obtain orthotropic laminates. However, the use of balanced stacks (a sufficient condition for membrane orthotropy) leads systematically to misleading solutions: whenever such a rule is used, bending orthotropy, a rather difficult property to achieve [21], is simply understated, assumed, but not really obtained [18, 22-24].

To overcome the previous restrictions, in the present study the multi-scale two-level (MS2L) optimisation approach based on the Verchery's polar method [25] for designing anisotropic complex structures [26-28] is used. In this background, the design problem is formulated in the most general sense, i.e. without introducing simplifying hypotheses and by considering, as design variables, the full set of geometric and mechanical parameters defining the behaviour of the laminate at each characteristic scale (mesoscopic and macroscopic).

In the context of the MS2L methodology, the optimisation problem is split in two distinct sub-problems. At the first level (macroscopic scale) the goal is to find the optimum value of both geometric and mechanical design variables of the laminate satisfying the design problem which is formulated in the form of a constrained non-liner programming problem (CNLPP). The second-level problem focuses on the laminate mesoscopic scale (i.e. the ply-level) and aims at finding at least one optimum stack meeting the geometrical and mechanical parameters resulting from the first-level problem.

The MS2L approach is based on the generalisation of the Verchery's polar method to the case of high-order equivalent single layer theories $[21,29,30]$ as well as on a GA previously developed by the first author [31, 32]. The MS2L optimisation strategy has already been successfully applied in the past to many real-world engineering problems [26-28, 33-38].

A rigorous experimental validation of such an approach is the main purpose of this study. In this work, the MS2L optimisation strategy is applied to the problem of minimising the mass of a moderately thick multilayer plate subject to requirements on both in-plane overall stiffness and first buckling load. The numerical work is complemented by an experimental campaign that aims not only at validating the MS2L optimisation approach, but also the effectiveness of the very general stacking sequences resulting from the process, as well as the influence of the transverse shear stiffness on the optimum solution (that cannot be neglected for moderately thick laminate) and which can be easily integrated in the framework of the polar method.

The paper is organised as follows: an overview of both numerical and experimental activities, followed by the description of the selected optimisation problem and the MS2L strategy is given in Sec. 2. The mathematical formulation of the first-level problem is detailed in Sec. 3, while the problem of determining suitable stacking sequences is formulated in Sec. 4. A concise description of the Finite Element (FE) model used in the optimisation process is given in Sec. 5, whilst the details about the experimental activities can be found in Sec. 6. The comparison between numerical and experimental results is presented in Sec. 
7, while Sec. 8 ends the paper with some concluding remarks.

\section{Multi-scale optimisation of composite structures: fundamental aspects}

\subsection{General work-flow}

The work-flow of the activities described in this study is shown in Fig. 1.

A representative design case is considered: the least-weight design of a multilayer composite plate subject to constraints on both the first buckling load (under uni-axial compressive load) and on the overall membrane stiffness. Such requirements are derived from their counterparts evaluated for a reference laminate (whose stack is built by using the classical rules taken from literature) having the same geometry of the optimised plate and subjected to the same boundary conditions (more details on the problem formulation at each scale are given in Secs.3 and 4).

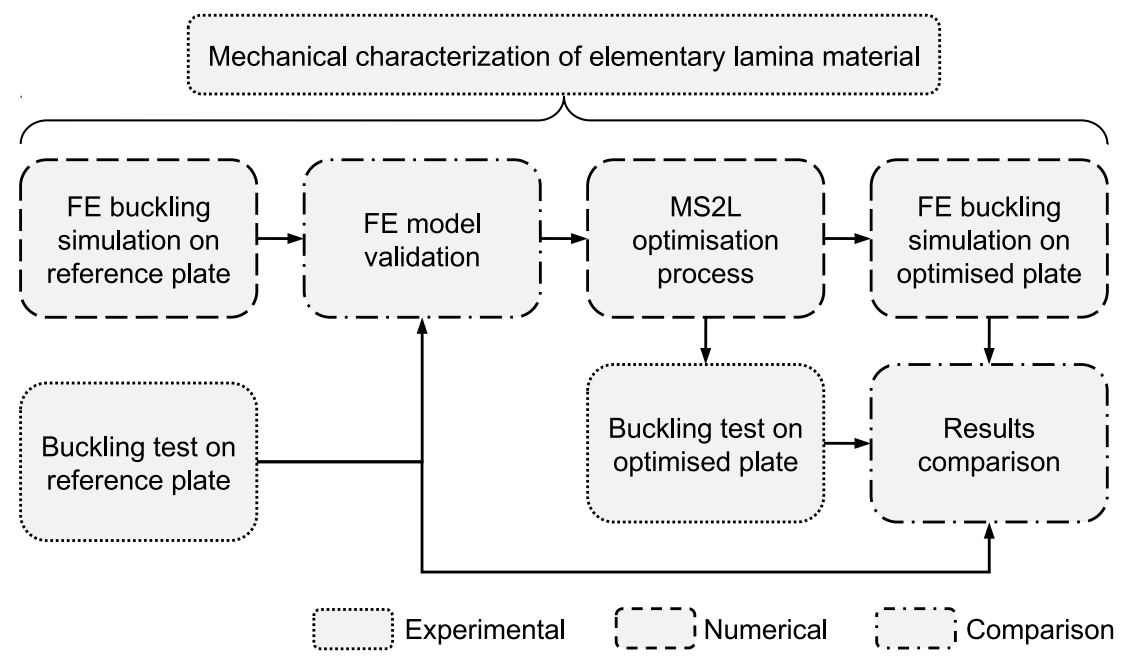

Figure 1: Numerical-experimental work-flow.

With the aim of experimentally validating the MS2L optimisation strategy, numerical and experimental activities proceed simultaneously and interact at given moments:

- the FE model is created and its geometry and mesh properly parametrized in terms of the design variables for the problem at hand (Secs. 3 and 5);

- a reference plate is fabricated and tested (Sec. 6);

- experimental results on the reference plate are compared to those provided by the FE model, to validate the latter (Sec. 6);

- the MS2L optimisation strategy is run by using the validated FE model to obtain the optimised plate stacking sequence (Secs. 3, 4 and 5);

- the optimum plate is fabricated and tested (Sec. 7);

- experimental results on the optimum solution are compared to the numerical ones in order to give an experimental proof of the effectiveness of the stacking sequence selected by the optimisation process as well as that of the MS2L optimisation strategy itself. 
All the above steps require the elastic properties of the constitutive lamina, so they have been preceded by a characterisation tests campaign.

\subsection{Problem Description}

The optimisation strategy presented in this study is applied to a simple composite structure, whose geometry and size are illustrated in Fig. 2. The specimen illustrated in Figs. 2 and 3 is a rectangular multilayer composite plate with two resin blocks at its ends that facilitate the loading in compression.

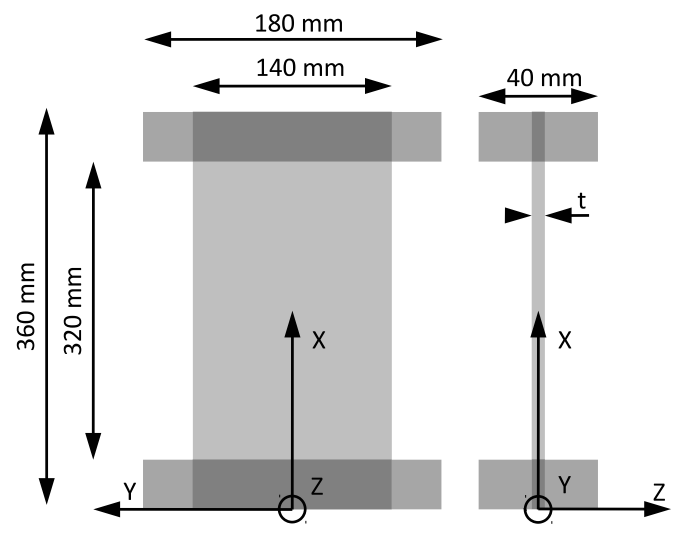

Figure 2: Geometry and overall size of the multilayer composite plate.
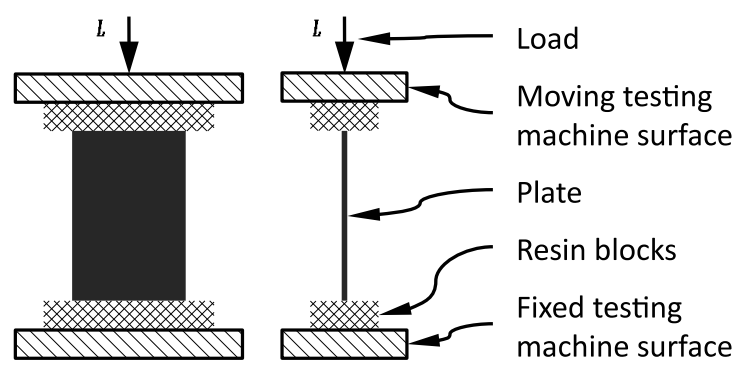

Figure 3: Simplified loading scheme of the multilayer composite plate.

The blocks are made of epoxy resin Araldite ${ }^{\circledR}$ LY $5052 /$ Aradur ${ }^{\circledR} 5052$ while the multilayer plate is made of the carbon/epoxy pre-preg HexPly ${ }^{\circledR}$ M21/34\%/UD194/IMA-12K by Hexcel ${ }^{\circledR}$. The elastic properties of both materials are reported in Tables 1 and 2, respectively.

Table 1: Material properties of the epoxy resin Araldite ${ }^{\circledR}$ LY5052-Aradur ${ }^{\circledR} 5052$ when considering a curing cycle of two hours at $60^{\circ} \mathrm{C}$ plus six hours at $80^{\circ} \mathrm{C}$.

\begin{tabular}{ll}
\hline Technical constants & Value \\
\hline$E[\mathrm{MPa}]$ & 3103.0 \\
$\nu$ & 0.35 \\
\hline
\end{tabular}

The material considered in this work is a pre-preg commonly used for aeronautical applications which is composed of unidirectional (UD) intermediate-modulus carbon-fibres (IMA-12k) and epoxy-matrix (M21, 34\% in weight) supplied in $300 \mathrm{~mm}$ wide rolls. 
Table 2: Material properties of the carbon/epoxy pre-preg HexPly ${ }^{\circledR}$ M21/34\%/ UD194/ IMA-12K by Hexcel ${ }^{\circledR}$

\begin{tabular}{|c|c|c|c|c|c|}
\hline \multicolumn{2}{|c|}{ Technical constants } & \multicolumn{2}{|c|}{ Polar parameters ${ }^{*}$ of $[\mathrm{Q}]^{a}$} & \multicolumn{2}{|c|}{ Polar parameters of $[\hat{\mathrm{Q}}]^{b}$} \\
\hline$E_{1}[\mathrm{MPa}]$ & 171500.0 & $T_{0}[\mathrm{MPa}]$ & 21687.9918 & $T[\mathrm{MPa}]$ & 4606.1923 \\
\hline$E_{1}^{c}[\mathrm{MPa}]$ & 146000.0 & $T_{1}[\mathrm{MPa}]$ & 20160.7437 & $R[\mathrm{MPa}]$ & 1275.8077 \\
\hline$E_{2}[\mathrm{MPa}]$ & 8659.0 & $R_{0}[\mathrm{MPa}]$ & 15805.9918 & $\Phi$ [deg] & 90.0 \\
\hline$G_{12}[\mathrm{MPa}]$ & 5882.0 & $R_{1}[\mathrm{MPa}]$ & 17275.5135 & & \\
\hline$\nu_{12}$ & 0.3245 & $\Phi_{0}[\mathrm{deg}]$ & 0.0 & & \\
\hline$\nu_{23}$ & 0.3 & $\Phi_{1}[\operatorname{deg}]$ & 0.0 & & \\
\hline \multicolumn{6}{|c|}{ Density and thickness } \\
\hline$\rho\left[\mathrm{Kg} / \mathrm{mm}^{3}\right]$ & \multicolumn{5}{|c|}{$1.58 \times 10^{-6}$} \\
\hline$t_{\text {ply }}[\mathrm{mm}]$ & \multicolumn{5}{|c|}{0.1831} \\
\hline
\end{tabular}

It is noteworthy that the elastic properties of the constitutive lamina, listed in Table 2, have been determined as a result of an experimental characterisation tests campaign, conducted at I2M laboratory in Bordeaux. Tests have been performed on thin laminated strips (having a length of $250 \mathrm{~mm}$ and a width of $20 \mathrm{~mm}$ ) with $\left[0^{\circ}\right]_{8},\left[90^{\circ}\right]_{8}$ and $\left[\left( \pm 45^{\circ}\right)_{2}\right]_{S}$ stacking sequences. The tests on $\left[\left( \pm 45^{\circ}\right)_{2}\right]_{S}$ specimens respect the ASTM D3518, D3518M94(07) standard [39]. Furthermore, the compressive Young's modulus along $x_{1}$-axis, $E_{1}^{c}$, listed in Table 2 has been taken directly from the manufacturer data-sheet: this quantity will be used to define the behaviour of the constitutive lamina within the FE model of the composite plate in order to carry out the calculation of the first buckling load as detailed in Sec. 5. More details on the whole campaign of characterisation tests can be found in [40].

The fundamental hypotheses about the macroscopic mechanical response of the structure focus essentially on the laminate behaviour:

- the material of the constitutive layer has a linear elastic transverse isotropic behaviour;

- the laminate is quasi-homogeneous and fully orthotropic [28, 30, 36, 37];

- at the macroscopic scale the elastic response of the laminate is described in the framework of the first order shear deformation theory (FSDT) and the stiffness matrices of the plate are expressed in terms of the laminate polar parameters [21, 29];

- no delamination occurs neither at the interfaces between the plies, nor at the interfaces between the plate and the resin blocks (perfect bonding condition).

Finally, it must be noticed that no simplifying hypotheses are made during the optimisation process on the stacking sequence of the multilayer plate, neither in terms of plies orientation angles nor in terms of the nature of the stack. Only avoiding the utilisation of $a$ priori assumptions that extremely shrink the solution space (e.g. the utilisation of symmetric, balanced stacks to attain membrane/bending uncoupling and membrane orthotropy, respectively) one can hope to obtain the true global optimum for a given problem: this is a key-point in the proposed approach. 


\subsection{Description of the multi-scale two-level optimisation strategy}

The main goal of the MS2L optimisation strategy is the least-weight design of the multilayer plate subject to constraints of different nature, i.e. mechanical, geometrical as well as feasibility constraints. The optimisation procedure is articulated into the following two distinct (but related) optimisation problems.

First-level problem. The aim of this phase (which focuses on the laminate macroscopic scale) is the determination of the optimum value of both mechanical and geometric parameters of the laminate in order to minimise its weight and to satisfy, simultaneously, the full set of imposed requirements (formulated as optimisation constraints). At this level the multilayer plate is modelled as an equivalent homogeneous anisotropic plate whose behaviour is described in terms of the laminate polar parameters [21, 29]. Therefore, the design variables of this phase are both the geometric and the polar parameters of the laminate.

Second-level problem. The second level of the strategy focuses on the laminate mesoscopic scale and aims at determining a suitable lay-up meeting the optimum combination of polar and geometric parameters of the laminate provided by the first-level problem. The goal is, hence, to find at least one stacking sequence which has to be quasi-homogeneous, fully orthotropic and that has to satisfy the optimum values of the polar parameters resulting from the first step. At this level of the strategy, the design variables are the layer orientations.

\section{Mathematical formulation of the first-level problem}

The macroscopic features of the composite have to be optimised during this phase. In particular, the mass minimisation of the laminate is here performed by satisfying, simultaneously, the set of optimisation constraints listed below:

1. a constraint on the first buckling load of the laminate (the laminate is subjected to a uni-axial compressive load);

2. a constraint on the membrane stiffness along the (in-plane) axis orthogonal to the applied load direction;

3. a feasibility constraint on the laminate polar parameters.

These aspects are detailed in the following subsections.

\subsection{The design variables}

The design variables for the problem at hand are of two types: geometrical and mechanical.

The only geometrical variable characterising the laminate at the macroscopic scale is its overall thickness $t$. Of course, $t$ is considered as a discrete optimisation variable having a step equal to the thickness of the elementary layer, i.e. $\Delta t=t_{\mathrm{ply}}$.

As far as the mechanical design variables are concerned, the macroscopic mechanical response of the laminate is described in the mathematical framework of the FSDT [41]. In this background, the constitutive law of the laminate (expressed within its global frame $R=\{0 ; x, y, z\})$ can be stated as:

$$
\left\{\begin{array}{c}
\{\mathrm{N}\} \\
\{\mathrm{M}\}
\end{array}\right\}=\left[\begin{array}{cc}
{[\mathrm{A}]} & {[\mathrm{B}]} \\
{[\mathrm{B}]} & {[\mathrm{D}]}
\end{array}\right]\left\{\begin{array}{c}
\left\{\varepsilon_{0}\right\} \\
\left\{\chi_{0}\right\}
\end{array}\right\},
$$


$\{\mathrm{F}\}=[\mathrm{H}]\left\{\gamma_{0}\right\}$,

where $[\mathrm{A}],[\mathrm{B}]$ and $[\mathrm{D}]$ are the membrane, membrane/bending coupling and bending stiffness matrices of the laminate, while $[\mathrm{H}]$ is the out-of-plane shear stiffness matrix. $\{\mathrm{N}\}$, $\{\mathrm{M}\}$ and $\{\mathrm{F}\}$ are the vectors of membrane forces, bending moments and shear forces per unit length, respectively, whilst $\left\{\varepsilon_{0}\right\},\left\{\chi_{0}\right\}$ and $\left\{\gamma_{0}\right\}$ are the vectors of in-plane strains, curvatures and out-of-plane shear strains of the laminate middle plane, respectively, [41].

In order to analyse the elastic response of the multilayer structure, the best practice consists in introducing the laminate normalised stiffness matrices:

$$
\begin{aligned}
& {\left[\mathrm{A}^{*}\right]=\frac{1}{t}[\mathrm{~A}],} \\
& {\left[\mathrm{B}^{*}\right]=\frac{2}{t^{2}}[\mathrm{~B}],} \\
& {\left[\mathrm{D}^{*}\right]=\frac{12}{t^{3}}[\mathrm{D}],} \\
& {\left[\mathrm{H}^{*}\right]= \begin{cases}\frac{1}{t}[\mathrm{H}] & \text { (basic), } \\
\frac{12}{5 t}[\mathrm{H}] & \text { (modified). }\end{cases} }
\end{aligned}
$$

As discussed in [21, 29], in the framework of the polar formalism it is possible to express the Cartesian components of these matrices in terms of their elastic invariants. It can be proven that, in the FSDT framework, for a fully orthotropic, quasi-homogeneous laminate (i.e. a laminate having the same orthotropic behaviour in terms of normalised membrane and bending stiffness matrices and whose membrane/bending coupling stiffness matrix is null) the overall number of independent mechanical design variables describing its mechanical response reduces to only three: the anisotropic polar parameters $R_{0 K}^{A^{*}}$ and $R_{1}^{A^{*}}$ and the polar angle $\Phi_{1}^{A^{*}}$ (this last representing the orientation of the main orthotropy axis) of matrix $\left[\mathrm{A}^{*}\right]$. For more details on the polar formalism and its application in the context of the FSDT the reader is addressed to [21, 29, 42].

In addition, in the formulation of the optimisation problem for the first level of the strategy, the feasibility constraints on the polar parameters (which arise from the combination of the layers orientations and positions within the stack) must also be considered. These constraints ensure that the optimum values of the polar parameters resulting from the first-level problem describe a feasible laminate that will be designed during the second step of the MS2L strategy, see [43]. Since the laminate is quasi-homogeneous, such constraints can be written only for matrix $\left[\mathrm{A}^{*}\right]$ :

$$
\left\{\begin{array}{c}
-R_{0} \leq R_{0 K}^{A^{*}} \leq R_{0}, \\
0 \leq R_{1}^{A^{*}} \leq R_{1}, \\
2\left(\frac{R_{1}^{A^{*}}}{R_{1}}\right)^{2}-1-\frac{R_{0 K}^{A^{*}}}{R_{0}} \leq 0 .
\end{array}\right.
$$

In Eq. (4), $R_{0}$ and $R_{1}$ are the anisotropic moduli of the ply reduced stiffness matrix [21].

It is noteworthy that, for the problem at hand, the main orthotropy direction of the laminate can be set equal to zero, i.e. $\Phi_{1}^{A^{*}}=0$ : this means that the main orthotropy axis 
is aligned with the direction of the applied load. In this way $\Phi_{1}^{A^{*}}$ is no longer a design variable.

For optimisation purposes it is useful to introduce dimensionless design variables. The dimensionless quantity related to the laminate overall thickness is, of course, the plies number $n$, while the dimensionless laminate polar parameters can be obtained by considering the ratio between the polar parameters of matrix $\left[\mathrm{A}^{*}\right]$ and the lamina counterparts. Therefore, the dimensionless laminate design variables can be defined as follows:

$$
n=\frac{t}{t_{\mathrm{ply}}}, \rho_{0}=\frac{R_{0 K}^{A^{*}}}{R_{0}}, \rho_{1}=\frac{R_{1}^{A^{*}}}{R_{1}} .
$$

In this background, Eq. (4) becomes:

$$
\left\{\begin{array}{c}
-1 \leq \rho_{0} \leq 1, \\
0 \leq \rho_{1} \leq 1, \\
2\left(\rho_{1}\right)^{2}-1-\rho_{0} \leq 0 .
\end{array}\right.
$$

Therefore, the dimensionless design variables defined above can be grouped into the vector of design variables:

$$
\xi^{\mathrm{T}}=\left\{n, \rho_{0}, \rho_{1}\right\} .
$$

First and second constraints of Eq. (6) can be taken into account as admissible intervals for the relevant optimisation variables, i.e. on $\rho_{0}$ and $\rho_{1}$. Thus, the resulting feasibility constraint on the laminate dimensionless polar parameters becomes:

$$
g_{1}(\boldsymbol{\xi})=2\left(\rho_{1}\right)^{2}-1-\rho_{0} \leq 0 .
$$

For a wide discussion upon the laminate feasibility and geometrical bounds as well as on the importance of the quasi-homogeneity assumption the reader is addressed to [43].

Finally, it must be noted that the laminate dimensionless polar parameters have to satisfy a further mechanical constraint related to the requirement on the membrane stiffness along the $y$-axis of the laminate global frame. This constraint can be states as:

$$
g_{2}(\boldsymbol{\xi})=1-\frac{A_{y y}(\boldsymbol{\xi})}{A_{y y}^{(\mathrm{ref})}} \leq 0,
$$

where $A_{y y}$ is the component of the membrane stiffness matrix along $y$-axis, while $A_{y y}^{(\mathrm{ref})}$ is its reference counterpart, i.e. the same quantity evaluated on the reference configuration of the laminate which is described in Sec. 6.

\subsection{Mathematical statement of the problem}

As previously stated, the aim of the first-level optimisation is the minimisation of the laminate mass by satisfying, simultaneously, constraints of different nature. 
In this context the optimisation problem can be formulated as a classical constrained non-linear programming problem (CNLPP):

$$
\min _{\boldsymbol{\xi}} \frac{M(\boldsymbol{\xi})}{M^{(\mathrm{ref})}}
$$

subject to:

$$
\left\{\begin{array}{l}
1-\frac{\lambda_{L}(\boldsymbol{\xi})}{\lambda_{L}^{(\text {ref })}} \leq 0, \\
g_{i}(\boldsymbol{\xi}) \leq 0, \text { with } i=1,2 .
\end{array}\right.
$$

The design space of the first-level problem, together with the type of each design variable, is detailed in Table 3. In Eq. (10) $M$ is the laminate mass, $\lambda_{L}$ is the first buckling load of the structure (calculated by means of an eigenvalue buckling analysis, see Sec 5), while $M^{(\mathrm{ref})}$ and $\lambda_{L}^{(\mathrm{ref})}$ are the counterparts for a reference solution (see Sec. 6) which is subject to the same boundary conditions (BCs) as those applied to the multilayer plate that will be optimised.

Table 3: Design space of the first-level problem.

\begin{tabular}{lllll}
\hline Design variable & Type & Lower bound & Upper bound & Discretisation step \\
\hline$n$ & integer & 16 & 32 & 1 \\
$\rho_{0}$ & continuous & -1.0 & 1.0 & - \\
$\rho_{1}$ & continuous & 0 & 1.0 & - \\
\hline
\end{tabular}

\subsection{Numerical strategy}

Problem (10) is a non-convex CNLPP in terms of both geometrical and mechanical variables. Its non-linearity and non-convexity is due on the nature of the buckling load constraint that is a non-convex function. In addition, the complexity of such a problem is also due to the non-linear feasibility constraints on the laminate polar parameters.

The total number of design variables at the laminate macroscopic scale is three. The number of optimisation constraints is three too (see Eq. (10)). Furthermore, design variables have different nature (see Table 3 ): integer $(n)$ and continuous $\left(\rho_{0}\right.$ and $\left.\rho_{1}\right)$ variables are involved in the definition of this CNLPP.

For the resolution of problem (10) the GA ERASMUS (EvolutionaRy Algorithm for optimiSation of ModUlar Systems) [32, 44] coupled with the FE model of the laminate (for calculating the first buckling load of the structure) has been utilised as optimisation tool for the solution search, see Fig. 4. The GA ERASMUS was already successfully applied to solve different kinds of real-world engineering problems, see for example [28, 33-37, 45, 46].

As shown in Fig. 4, for each individual at each generation, the numerical tool performs a $\mathrm{FE}$ analysis to calculate the first buckling load (eigenvalue problem) of the multilayer plate as well as its weight. The input data of the FE model of the composite structure (implemented in ANSYS ${ }^{\circledR}$ environment) are both geometrical and mechanical design variables (generated by ERASMUS). The GA elaborates the results provided by the FE model in order to execute the genetic operations. These operations are repeated until the GA meets the user-defined convergence criterion.

The generic individual of the GA ERASMUS represents a potential solution for the problem at hand. The genotype of the individual for problem (10) is characterised by only one chromosome composed of three genes, each one coding a component of the vector of design variables, see Eq. (7). 


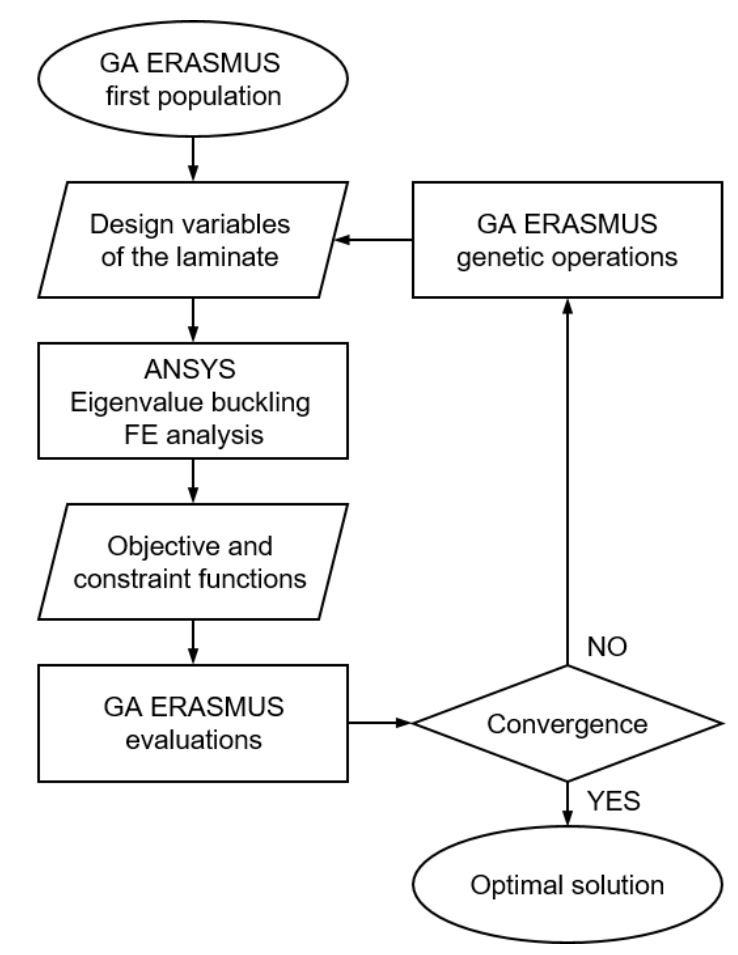

Figure 4: Logical flow of the numerical procedure for the solution search of the first-level problem.

\section{Methematical formulation of the second-level problem}

The second-level problem focuses on the laminate lay-up design. The goal is to determine at least one stacking sequence satisfying the optimum values of both geometric and polar parameters resulting from the first level of the strategy and having the elastic symmetries imposed to the laminate within the formulation of the first-level problem, i.e. quasi-homogeneity and orthotropy. In the framework of the FSDT (and when considering the polar formalism for representing the laminate stiffness matrices) this problem can be stated in the form of an unconstrained minimisation problem [21, 29]:

$$
\min _{\boldsymbol{\delta}} I\left(f_{i}(\boldsymbol{\delta})\right)
$$

with

$$
I\left(f_{i}(\boldsymbol{\delta})\right)=\sum_{i=1}^{6} f_{i}(\boldsymbol{\delta}) .
$$

where $\boldsymbol{\delta} \in \mathbb{R}^{n}$ is the vector of the layer orientations, i.e. the design variables of this phase, while $f_{i}(\boldsymbol{\delta})$ are quadratic functions in the space of polar parameters, each one representing a requirement to be satisfied, such as orthotropy, uncoupling, etc. For the problem at hand the partial objective functions can be written as:

$$
\begin{gathered}
f_{1}(\boldsymbol{\delta})=\left(\frac{\left|\Phi_{0}^{A^{*}}(\boldsymbol{\delta})-\Phi_{1}^{A^{*}}(\boldsymbol{\delta})\right|}{\pi / 4}-K^{A^{*}(o p t)}\right)^{2}, \quad f_{2}(\boldsymbol{\delta})=\left(\frac{R_{0}^{A^{*}}(\boldsymbol{\delta})-R_{0}^{A^{*}(o p t)}}{R_{0}}\right)^{2}, \\
f_{3}(\boldsymbol{\delta})=\left(\frac{R_{1}^{A^{*}}(\boldsymbol{\delta})-R_{1}^{A^{*}(o p t)}}{R_{1}}\right)^{2}, \quad f_{4}(\boldsymbol{\delta})=\left(\frac{\left|\Phi_{1}^{A^{*}}(\boldsymbol{\delta})-\Phi_{1}^{A^{*}(o p t)}\right|}{\pi / 4}\right)^{2}, \quad f_{5}(\boldsymbol{\delta})=\left(\frac{\|\mathbf{C}(\boldsymbol{\delta})\|}{\|\mathbf{Q}\|}\right)^{2}, \\
f_{6}(\boldsymbol{\delta})=\left(\frac{\left\|\mathbf{B}^{*}(\boldsymbol{\delta})\right\|}{\|\mathbf{Q}\|}\right)^{2},
\end{gathered}
$$


where $f_{1}(\boldsymbol{\delta})$ represents the elastic requirement on the orthotropy of the laminate having the prescribed shape (imposed by the value of $K^{A^{*}}$ provided by the first step of the procedure), $f_{2}(\boldsymbol{\delta}), f_{3}(\boldsymbol{\delta})$ and $f_{4}(\boldsymbol{\delta})$ are the requirements related to the prescribed values of the optimal polar parameters resulting from the first-level problem, while $f_{5}(\boldsymbol{\delta})$ and $f_{6}(\boldsymbol{\delta})$ are linked to the quasi-homogeneity condition.

$I\left(f_{i}(\boldsymbol{\delta})\right)$ is a positive, semi-definite, convex function in the space of the laminate polar parameters, see Eqs. (12)-(13). Nevertheless, such a function is highly non-convex in the space of laminae orientations because the laminate polar parameters depend upon circular functions of these angles. Moreover, the absolute minima of $I\left(f_{i}(\boldsymbol{\delta})\right)$ are known a priori since they are the zeroes of this function. For more details about the nature of the secondlevel problem, see [21, 29].

In order to simplify the problem of retrieving an optimum stack, the search space for problem (11) has been restricted to a particular class of quasi-homogeneous laminates: the quasi-trivial (QT) stacking sequences which constitute exact solutions with respect to the requirements of quasi-homogeneity, i.e. functions $f_{5}(\boldsymbol{\delta})$ and $f_{6}(\boldsymbol{\delta})$ in Eq. (13) are identically null for QT stacks.

QT solutions can be found for laminates with identical plies by acting only on the position of the layers within the stack. Indeed, QT stacks are exact solutions, in terms of quasi-homogeneity condition, regardless of the value of the orientation angle assigned to each layer. In this way, orientations represent free parameters which can be optimised to fulfil further elastic requirements, i.e. functions $f_{1}(\boldsymbol{\delta}), f_{2}(\boldsymbol{\delta}), f_{3}(\boldsymbol{\delta})$ and $f_{4}(\boldsymbol{\delta})$.

The procedure for searching QT stacks is conceptually simple. Let $n$ be the number of layers and $n_{g} \leq n$ the number of saturated groups [47]. Plies belonging to a given saturated group share the same orientation angle $\theta_{j},\left(j=1, \ldots, n_{g}\right)$. The idea is to look for all the permutations of the position of the plies indexes belonging to each group which meet the quasi-homogeneity condition. More details on this topic can be found in [47].

Suppose now to fix both the number of plies and of saturated groups, namely $n$ and $n_{g}$. As discussed in [47], the problem of determining QT stacks for a given couple of $n$ and $n_{g}$ can give rise to a huge number of solutions: the number of QT stacks rapidly increases along with $n$. To this purpose a database of QT stacks has been built for different combinations of $n$ and $n_{g}$.

For the problem at hand, the optimum number of plies $n$ constitutes a result of the first-level problem, while the number of saturated groups $n_{g}$ has been fixed a priori. Let $n_{\text {sol }}$ be the number of QT stacks for a particular combination of $n$ and $n_{g}$. Each solution collected within the database is uniquely defined by means of an identifier $\operatorname{ID}_{\text {sol }}$ (i.e. an integer) which varies in the range $\left[1, n_{\text {sol }}\right]$. Therefore, $\mathrm{ID}_{\text {sol }}$ represents a further design variable along with the $n_{g}$ orientation angles of different saturated groups, i.e. $\boldsymbol{\theta} \in \mathbb{R}^{n_{g}}$. The design variables can be thus collected into the following vector,

$$
\boldsymbol{\eta}^{T}=\left\{\mathrm{ID}_{\mathrm{sol}}, \theta_{1}, \ldots, \theta_{n_{g}}\right\},
$$

and problem (11) can be reformulated as

$$
\min _{\boldsymbol{\eta}} \sum_{i=1}^{4} f_{i}(\boldsymbol{\eta}),
$$

$f_{5}(\boldsymbol{\eta})$ and $f_{6}(\boldsymbol{\eta})$ being identically null. 
In this background, the solution search for problem (15) is performed by means of the GA ERASMUS. In the case of QT stacks the structure of the individual genotype is simple because it is composed of a single chromosome with $n_{g}+1$ genes: the first one codes the variable $\mathrm{ID}_{\text {sol }}$ whilst the remaining genes code the orientation angles of every saturated group which are discrete variables in the range $\left[-89^{\circ}, 90^{\circ}\right]$ with a step length equal to $1^{\circ}$.

\section{Finite element model of the multilayer plate}

The FE model of the multilayer plate involved in the first level of the MS2L strategy is built using the FE commercial code ANSYS ${ }^{\circledR}$. As far as the optimisation process is concerned, a linear eigenvalue buckling analysis is conducted to determine the value of the first buckling load for each individual, i.e. for each point in the design space, at the current generation.

The need to analyse, within the same generation, different geometrical configurations (laminates with different geometrical and mechanical properties), each one corresponding to an individual, requires the creation of an ad-hoc input file for the FE code that has to be interfaced with ERASMUS. The FE model must be conceived to account for variable geometry, material and mesh. Indeed, for each individual at the current generation, the FE code has to be able to vary such quantities, thus a proper parametrisation of the model has to be done.

The FE model of the laminate is illustrated in Fig. 5. The model has been built by using a combination of four-nodes shell elements with six Degrees Of Freedom (DOFs) per node (ANSYS SHELL181 elements), eight-nodes solid elements with three DOFs per node (ANSYS SOLID185 elements) and non-linear multi-point constraints elements (ANSYS MPC184 elements).

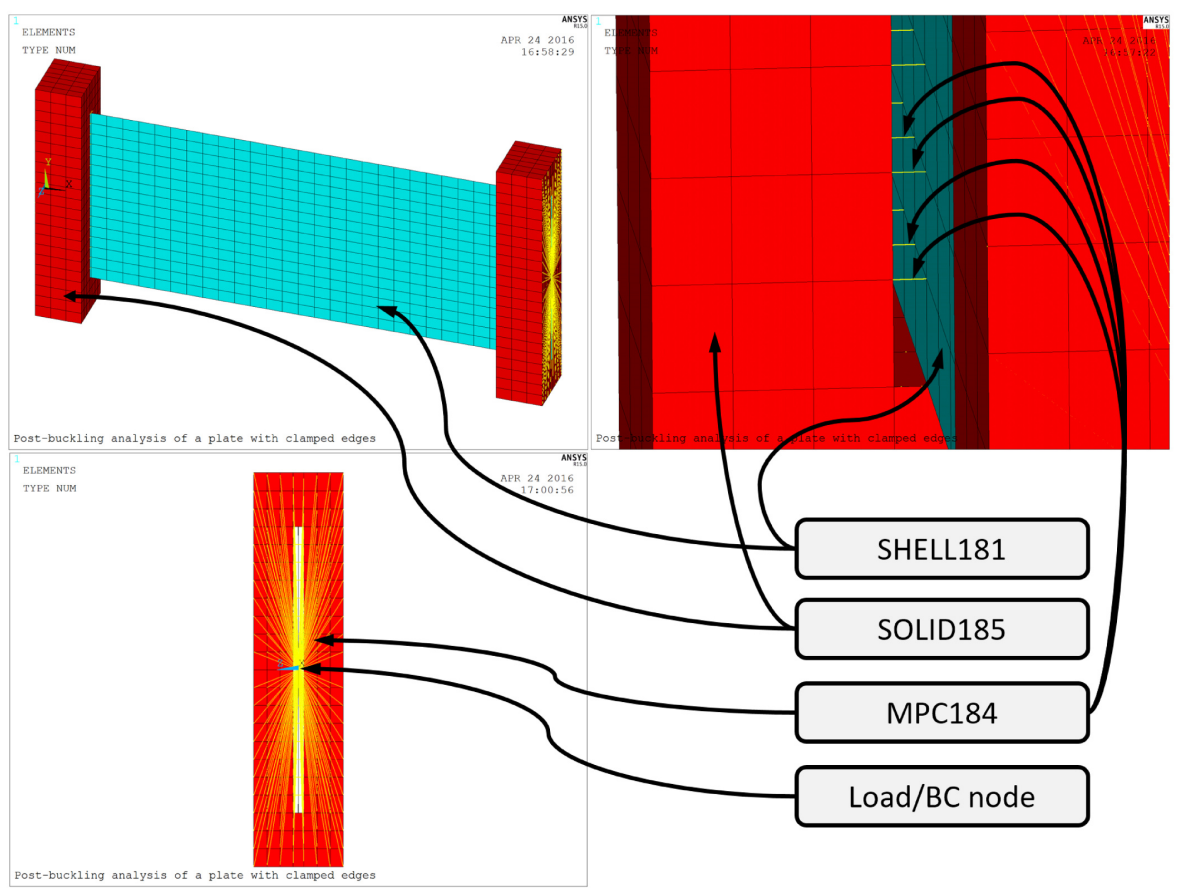

Figure 5: FE model of the multilayer composite plate and the details of MPC184 elements.

SHELL281 elements have been used to model the multilayer composite plate at the macroscopic scale, regardless of the stacking sequence, i.e. their mechanical behaviour is 
described by defining directly the homogenised stiffness matrices $\left[\mathrm{A}^{*}\right],\left[\mathrm{B}^{*}\right],\left[\mathrm{D}^{*}\right]$ and $\left[\mathrm{H}^{*}\right]$ (by using a pre-integrated definition of the shell section).

SOLID185 elements have been considered to model resin blocks (full integration with incompatible modes has been set), while the compatibility of the displacement field at the interface between the plate and the resin blocks is achieved through ANSYS MPC184 elements whose formulation is based upon a classical multi-point constraint element scheme [48]. MPC184 elements are defined between each couple of nodes belonging to contiguous shell and solid elements, as depicted in Fig. 5. In particular, MPC184 elements are defined between nodes of the middle plane of the multilayer plate (master nodes) and those belonging to the internal faces of the resin blocks (slave nodes).

Furthermore, MPC184 elements have been used to simulate the boundary conditions (BCs) imposed by the experimental apparatus (see Sec. 6). In particular, two pilot nodes, $\mathrm{A}=\{0,0,0\}$ and $\mathrm{B}=\{L, 0,0\}$, have been defined according to the structure global frame illustrated in Fig. 2 ( $L=360 \mathrm{~mm}$ is the overall length of the structure along $x$-axis). Then, nodes A and B have been connected (through MPC184 elements) to those located on the resin blocks end faces, i.e. faces located at $x=0$ and $x=L$, respectively (see Fig. 5 ). The $\mathrm{BCs}$ for nodes $\mathrm{A}$ and $\mathrm{B}$ are

$$
\begin{array}{ll}
\text { node A: } & u_{i}=0, \beta_{i}=0 ; \\
\text { node B: } & F_{x}=-1 \mathrm{~N}, u_{y}=u_{z}=0, \beta_{i}=0,
\end{array}
$$

$(i=x, y, z)$.

In Eq. (16) $u_{i}$ and $\beta_{i}$ are nodal displacements and rotations, respectively, whilst $F_{x}$ is the $x$ component of the nodal force.

Concerning the comparison between numerical and experimental results, a non-linear buckling analysis has been performed on the FE model of the structure both on the reference structure (to validate the FE model it-self) and on the optimum configurations (for verification purposes). Let $\lambda_{L}$ be the value of the first buckling load resulting from the eigenvalue analysis and $\mathbf{u}_{L}(x, y, z)$ the corresponding eigenvector (i.e. the normalised displacement field of the structure). In order to carry out the non-linear buckling load analysis, the initial geometry of the multilayer plate is perturbed by introducing a fictitious geometrical imperfection. As usually done in these cases [48], the geometrical imperfection is simulated through a small perturbation of the nodes location by applying a scaling factor (equal to 0.1, resulting in a deformation of the same magnitude of the geometrical defects measured on the specimens) to the previous displacement field $\mathbf{u}_{L}(x, y, z)$. Only the Cartesian coordinates of the nodes are perturbed without introducing any additional stress/strain field. Subsequently, a non-linear static analysis is performed by applying to the pilot node B a compressive force $F_{x}=1.1 \lambda_{L}$ and by stopping the analysis when that same pilot node has reached a displacement $u_{x}=-0.8 \mathrm{~mm}$ (i.e. approximately the same displacement measured during the experimental test on the reference specimen, see Sec. 6). The non-linear buckling load $\lambda_{N L}$ is then computed as the last value of the reaction force along $x$-axis measured at the pilot node A. The arc-length method [48] has been chosen as a numerical technique to find the solution for the non-linear equilibrium problem.

Finally, before starting the optimisation process, a sensitivity study (not reported here for the sake of brevity) on the proposed FE model with respect to the mesh seed parameters $n_{x}^{\alpha}, n_{y}^{\alpha}, n_{z}^{\alpha},(\alpha=b, p$, depending on the region), illustrated in Fig. 6, has been conducted. It was observed that a mesh having $n_{x}^{b}=n_{y}^{b}=n_{z}^{b}=3, n_{x}^{p}=30, n_{y}^{p}=16$ and $n_{z}^{p}=1$ is sufficient to properly evaluate the first buckling load (both linear and non-linear) of the structure. 


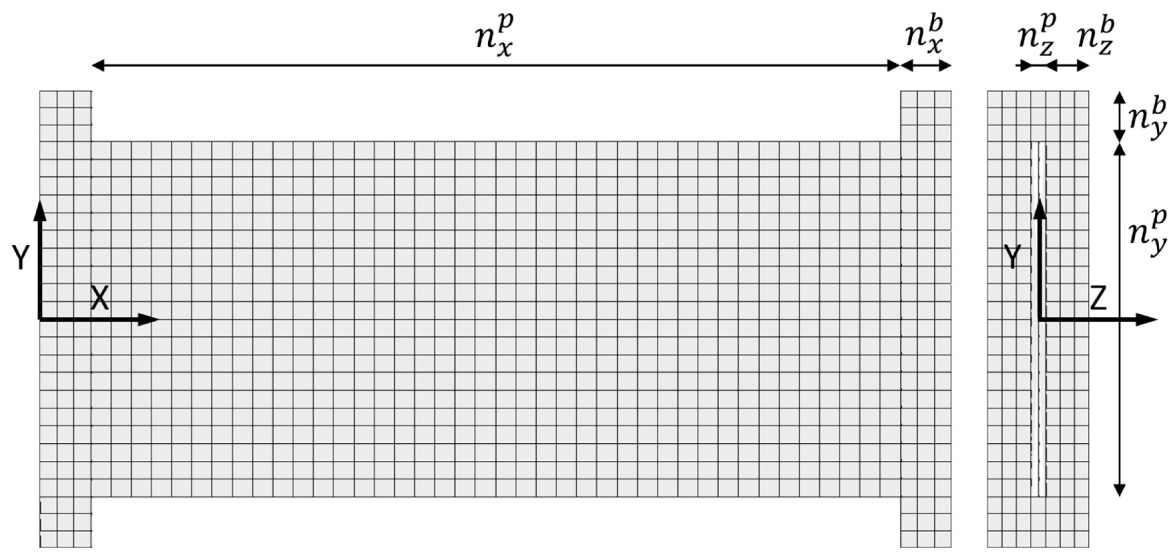

Figure 6: Mesh seed parameters for the FE model of the multilayer composite plate.

\section{Experimental apparatus for buckling analysis}

A schematic representation of the specimen geometry and loading condition is given in Figs. 2 and 3. The specimen reference system is shown in Fig. 2: the positive $z$-direction points from the plate surface which is in contact to the mould (during the curing cycle) towards the other one. The edges of the plate that are in touch with the testing machine are embedded into two resin blocks. On the one hand, these resin blocks allow for an easy placement and load application during the tests. On the other hand, they avoid the introduction of high local stresses on the plate edges which can lead to a premature failure of the specimen.

Two specimens are tested: one for the reference configuration and one among the different optimum solutions provided by the MS2L optimisation method (see Sec. 7). Each sample is obtained from a $420 \times 300 \mathrm{~mm}$ panel fabricated by computer numerical controlled (CNC) cutting and manual lay-up of the used pre-preg composite material and than cured following the guidelines given by the manufacturer. A rectangular plate has been obtained from the central part of each panel by means of a horizontal mill equipped with a circular diamond blade, then the two long edges of the plates have been lightly ground to improve their parallelism. To embed the plates into the resin blocks, some simple custom tools have been fabricated. The blocks are formed into a semi-rigid silicon mould obtained from an aluminium CNC machined mould. The frame shown in Fig. 7 has been created to keep in place the plate and the mould during the resin polymerisation process ensuring, in this way, a good and stable alignment. The so obtained specimens had some defects on their bottom and top surfaces: air bubbles coming from the polymerisation process as well as non-acceptable flatness and parallelism levels. Accordingly, by using one of the long edges of the plate as reference, the two block surfaces and the embedded ends of the plate have been milled.

Before performing the buckling tests, each specimen has undergone a series of measurements to evaluate its geometrical defects.

The frame illustrated in Fig. 7 has a double function: it acts as a positioning tool for the specimen as well as a support for the measuring sensors during each test. During the test various data have been collected (Fig. 8 and Fig. 9 show the typical testing set-up):

- longitudinal displacement $\left(u_{x}\right)$ and rotations $\left(\beta_{y}\right.$ and $\left.\beta_{z}\right)$ of the moving surface of the testing machine via three Linear Variable Displacement Transducer (LVDT) sensors; 


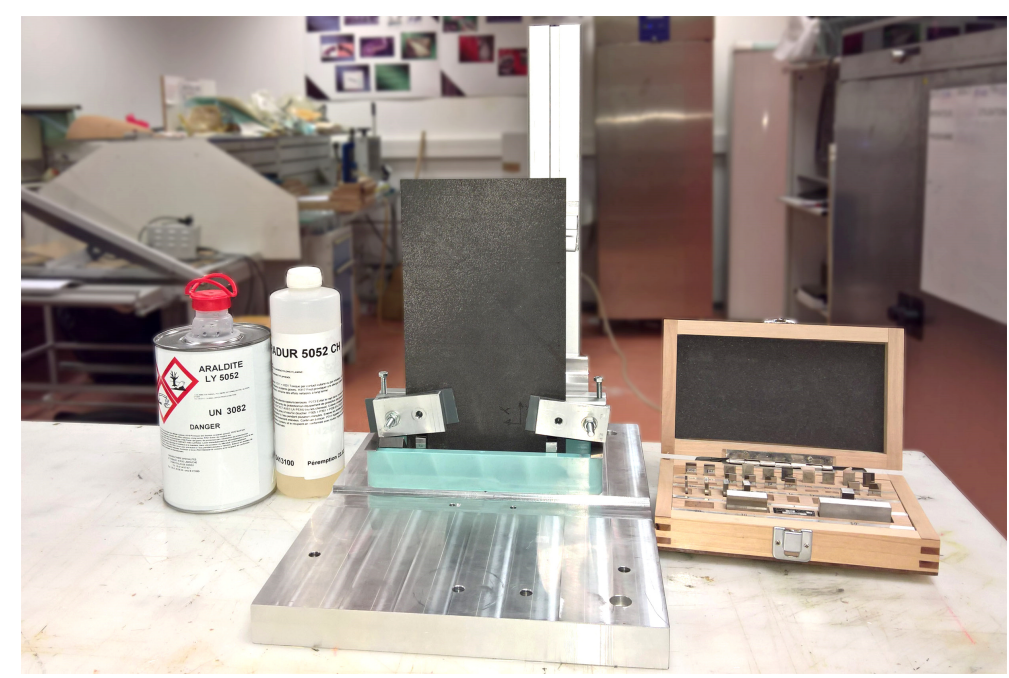

Figure 7: Curing assembly.

- out-of-plane displacement of the middle part of the longitudinal axis of the plate via a laser sensor;

- strain data via strain gages;

- the applied load via a load cell.

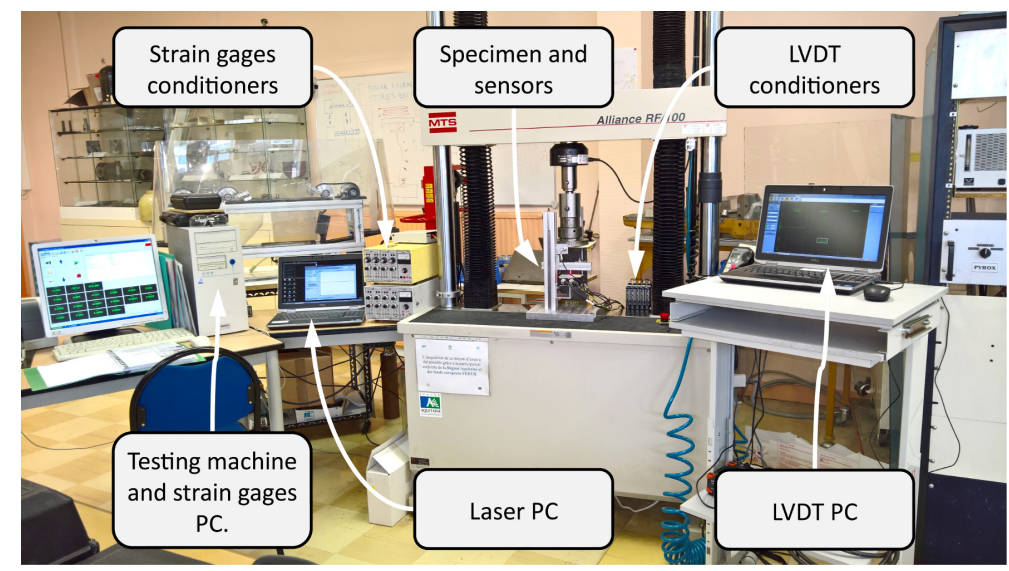

Figure 8: Testing set-up 1.

The specimens have been tested in an $\mathrm{MTS}^{\circledR}$ Alliance RF/100 high-performance electromechanical (EM) load frame equipped with flat fixed (non-rotating) surface heads. The data have been recorded using three PC-driven data acquisition systems and then combined via time-based synchronisation.

All tests have been performed at a fixed cross-head displacement rate of $0.2 \mathrm{~mm} / \mathrm{min}$ and stopped when reaching the given strain of $\varepsilon_{\max }=0.25 \%$ on one of the two back to back strain gages positioned at the center of the plate.

\subsection{The reference configuration: numerical vs. experimental results}

Before starting the multi-scale optimisation process, a reference configuration must be defined in order to establish reference values for the mass as well as for the first buckling 


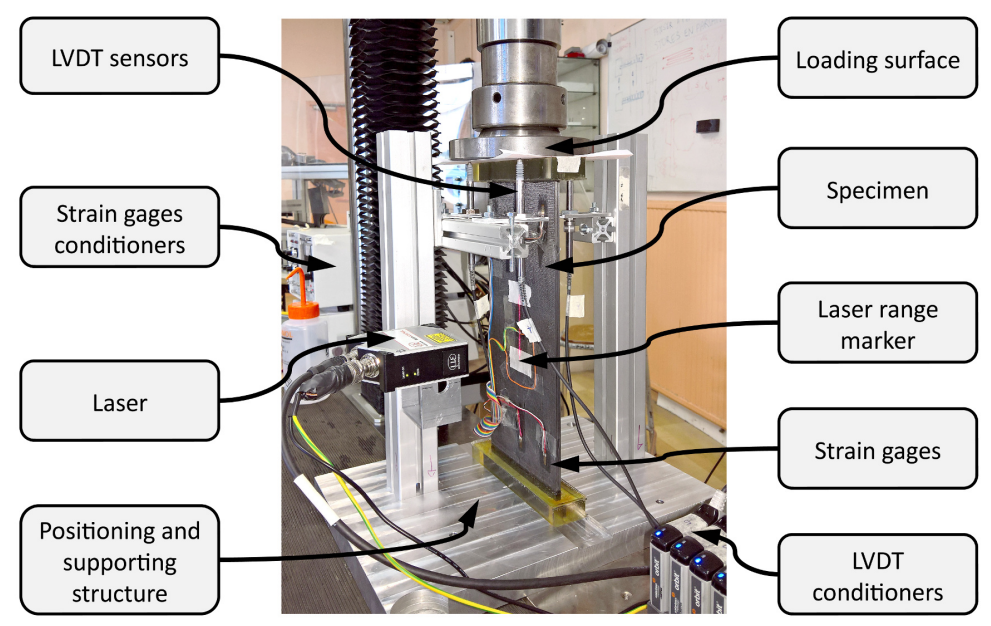

Figure 9: Testing set-up 2.

load and the membrane stiffness along $y$-axis. Of course, the reference multilayer plate has the same geometry (in plane overall size) and it is subject to the same set of BCs, as those applied on the specimen that will be optimised. As a reference solution a symmetric quasi-isotropic laminate composed of 32 layers has been chosen. The plies are arranged according to the following stack: $\left[\left(45^{\circ}, 0^{\circ},-45^{\circ}, 90^{\circ}\right)_{S}\right]_{4}$, i.e. the laminate is uncoupled and the membrane stiffness matrix is isotropic, but the bending one is totally anisotropic. This reference solution corresponds to a classical configuration utilised in the aeronautical field: its mass and its overall stiffness (both bending and membrane) still represent a "good" compromise between lightness and stiffness requirements.

For the purposes of this work, among the different data recorded during the tests, only the applied load versus longitudinal displacement has been considered. For the reference solution, this curve is illustrated in Fig. 10, where also its numerical counterpart (the buckling load resulting from both linear eignevalue and non-linear buckling analyses) provided by the FE model is plot for comparison purposes.

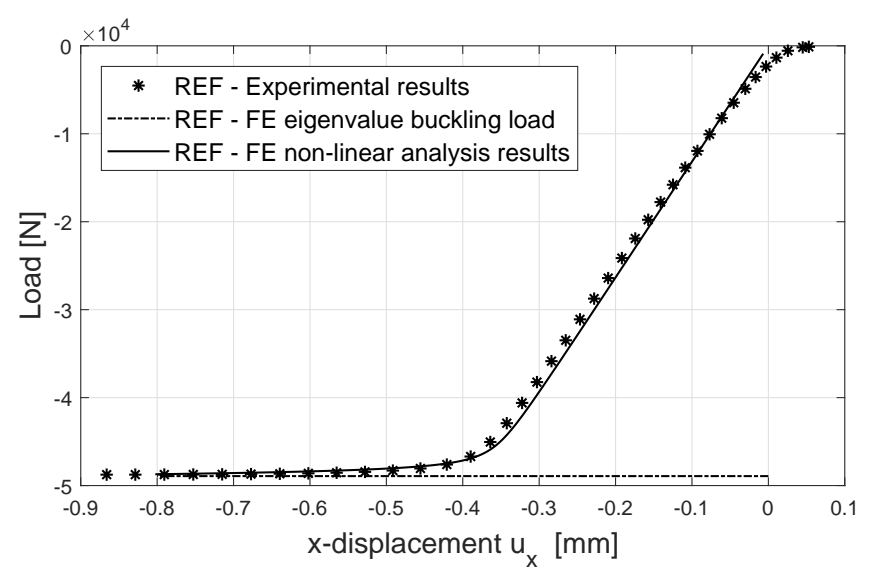

Figure 10: Experimental and numerical results comparison, reference solution.

A summary of the results on the reference solution is given in Table 4.

The numerical value of the buckling load ( $\lambda_{N L}$ from the non-linear simulation) shows a difference of $-0.1 \%$ compared to the experimental one, confirming in this way the excellent agreement between numerical and experimental results. 
Table 4: Numerical and experimental mechanical properties of the reference solution.

\begin{tabular}{l|r}
\hline Property & Reference solution \\
\hline$n_{p l y}$ & 32 \\
$A_{y y}[\mathrm{~N} / \mathrm{mm}]$ & 363326 \\
$\lambda_{(\exp )}[\mathrm{N}]$ & 48760 \\
$\lambda_{L}[\mathrm{~N}]$ & 48924 \\
$\lambda_{N L}[\mathrm{~N}]$ & 48712 \\
\hline
\end{tabular}

As far as the membrane stiffness along $y$-axis is concerned, this data has not been experimentally measured, but it is derived analytically according to the FSDT formulae.

\section{Optimum solutions: numerical and experimental results}

The genetic parameters tuning the behaviour of the GA used to perform the solution search, for both first and second-level problems, are listed in Table 5. Moreover, concerning the constraint-handling technique for the first-level problem, the Automatic Dynamic Penalisation (ADP) method has been considered, see [44]. For more details on the optimisation tool and the meaning of the values of the different parameters tuning the GA, the reader is addressed to [31].

For the first-level problem, the overall optimisation process has required approximately 7 days ( $6 \mathrm{~s}$ for each eigenvalue buckling analysis) using two cores of a machine with an Intel Xeon E5-2697v2 processor (2.70-3.50 GHz). The computational effort related to the second level problem requires only few seconds on the same machine.

The optimum values of both geometric and mechanical design variables (dimensionless variables) resulting from the first level of the optimisation strategy are listed in Table 6 . Since problem (10) is non-convex, many optimal solutions exist: only the first three best configurations have been reported in Table 6. These solutions exhibit the "best" compromise between lightness and the requirements on buckling and membrane stiffness. It is noteworthy that each optimum solution is composed of 29 plies, which means a weight saving of $9.4 \%$ when compared to the reference configuration of the multilayer plate. At the macroscopic scale, each one of the three laminates reported in Table 6 is quasi-homogeneous and fully orthtropic (both membrane and bending stiffness matrices) with an ordinary orthotropy shape (parameter $K^{A^{*}}=0$ because the dimensionless anisotropic polar modulus $\rho_{0}$ is positive, see [21]). For each solution, the dimensionless polar parameter $\rho_{1}$ is about an order of magnitude lower than $\rho_{0}$ : this means that the laminate tends to exhibit a square symmetric behaviour (for both membrane and bending stiffness matrices). For a deeper insight on these aspects, the interested reader is addressed to [21, 29].

As stated in Sec. 4, the second-level problem is solved in the space of QT stacks. After fixing the number of plies $n$ and the number of saturated groups $n_{g}$, the design variables are the identifier of the QT solution as well as the orientation angle of each saturated group, see Eq. (14). Because problem (15) is highly non-convex in the space of the orientation angles of saturated groups, it is possible to find multiple solutions (theoretically an infinite number) meeting the optimum value of the laminate polar parameters provided by the first-level problem. A non-exhaustive list of them, in the case of $n_{g}=5$, is presented in Table 7 and the respective mechanical properties (in terms of membrane stiffness along $y$-axis and buckling load resulting from the non-linear analysis) can be found in Table 8 .

It is noteworthy that all these solutions are non-standard stacks. Although such sequences are neither symmetric nor balanced, they are fully orthotropic (both in membrane 
Table 5: GA parameters used for the solution search of both first-level and second-level problems.

\begin{tabular}{lll}
\hline Property & First-level pb. & Second-level pb. \\
\hline N. of populations & 2 & 2 \\
N. of individuals per population $\left(N_{\text {ind }}\right)$ & 200 & 500 \\
N. of chromosomes & 1 & 1 \\
N. of genes & 3 & 3 \\
Stop criterion & Fixed generations $(250)$ & Fixed generations $(500)$ \\
Crossover probability & 0.85 & 0.85 \\
Mutation probability & $0.005\left(=1 / N_{\text {ind }}\right)$ & $0.002\left(=1 / N_{\text {ind }}\right)$ \\
Selection operator & Roulette wheel & Roulette wheel \\
Elitism operator & Active & Active \\
Isolation time & 10 & 20 \\
\hline
\end{tabular}

Table 6: Optimum solutions of the first-level problem.

\begin{tabular}{l|lll}
\hline \multirow{2}{*}{ ID } & \multicolumn{3}{|c}{ Design variables } \\
& $n$ & $\rho_{0}$ & $\rho_{1}$ \\
\hline 1.01 & 29 & 0.9863 & 0.0978 \\
1.02 & 29 & 0.9941 & 0.0821 \\
1.03 & 29 & 0.9589 & 0.0929 \\
\hline
\end{tabular}

and bending), uncoupled and they exhibit the same elastic behaviour (in terms of normalised membrane and bending stiffness). These peculiar features are the natural result of the utilisation of very general QT stacks. Furthermore, these optimum solutions really represent equivalent configurations: they share the same number of plies and they show light differencies in terms of mechanical performances. As reported in Table 8, these differencies range from $+1.7 \%$ to $+3.9 \%$ for the buckling load and from $+2.2 \%$ to $+3.3 \%$ for $A_{y y}$. Therefore, each optimum configuration is simultaneously lighter and stiffer than the reference one and this result has been achieved only by abandoning the usual engineering rules related to the nature of the stacking sequence.

Table 7: Numerical results of the second-level problem (optimum stacking sequences).

\begin{tabular}{llll}
\hline ID & Parent & Stacking Sequence \\
\hline 2.01 & 1.01 & {$[89 / 0 / 90 / 90 / 2 / 90 /-3 /-3 / 2 / 0 /-3 / 2 / 0 / 0 / 90 / 90 / 89 / 90 / 89 /-3 / 2 / 90 / 89 / 90 / 0 / 0 / 90 / 2 /-3]$} \\
2.02 & 1.01 & {$[-87 / 88 / 1 /-2 /-2 / 0 / 0 / 88 / 88 / 1 / 88 / 88 /-2 / 1 / 0 / 88 /-87 / 0 /-87 /-2 / 1 /-2 /-87 / 88 / 88 /-2 / 1 / 88 / 0]$} \\
2.03 & 1.01 & {$[4 /-2 / 0 / 89 / 89 /-89 /-89 /-89 /-2 /-2 /-89 / 0 / 89 /-2 / 0 / 0 / 4 /-2 / 4 / 89 /-89 / 89 / 4 /-2 /-89 / 89 /-89 /-2 / 0]$} \\
2.04 & 1.01 & {$[-87 / 88 /-2 / 1 / 1 / 88 /-1 / 88 /-2 / 88 /-1 / 1 /-1 / 1 /-2 / 88 /-87 / 1 /-87 / 88 / 88 /-2 /-87 / 1 / 88 /-2 / 88 / 1 /-1]$} \\
2.05 & 1.01 & {$[-89 /-2 /-1 / 90 / 90 /-2 / 6 / 90 /-1 / 90 / 6 /-2 / 6 / 90 /-1 /-2 /-89 / 90 /-89 /-2 /-2 /-1 /-89 / 90 / 90 /-1 / 90 /-2 / 6]$} \\
2.06 & 1.01 & {$[-89 / 90 / 1 / 90 /-4 / 4 /-4 / 1 / 4 / 4 / 1 / 90 /-89 /-89 / 90 /-4 / 90 / 1 /-4 / 90 / 90 /-89 / 4 / 1 /-89 / 1 / 4 /-4 / 90]$} \\
2.07 & 1.01 & {$[-89 / 90 / 3 / 0 / 0 /-2 /-2 / 90 / 90 / 3 / 90 / 90 / 0 / 3 /-2 / 90 /-89 /-2 /-89 / 0 / 3 / 0 /-89 / 90 / 90 / 0 / 3 / 90 /-2]$} \\
2.08 & 1.01 & {$[90 / 90 / 0 / 0 / 0 / 0 / 0 / 90 / 90 / 0 / 90 / 90 / 0 / 0 / 0 / 90 / 90 / 0 / 90 / 0 / 0 / 0 / 90 / 90 / 90 / 0 / 0 / 90 / 0]$} \\
2.09 & 1.01 & {$[-2 / 0 / 88 /-88 / 0 / 1 / 88 /-88 / 1 / 1 / 88 / 1 /-88 /-88 / 88 / 88 /-2 / 0 /-2 / 0 / 0 /-88 /-2 / 0 / 1 / 88 / 88 / 1 /-88]$} \\
2.10 & 1.02 & {$[-87 / 89 /-9 / 3 / 89 / 3 / 6 / 6 /-9 / 89 / 6 / 89 / 3 / 89 /-9 / 89 /-87 / 3 /-87 / 6 / 3 /-9 /-87 / 89 / 89 /-9 / 89 / 3 / 6]$} \\
2.11 & 1.02 & {$[8 /-7 / 88 / 88 / 3 /-87 /-87 /-7 / 3 /-7 / 88 / 3 / 88 /-7 /-87 / 88 / 8 /-87 / 8 /-7 / 3 / 88 / 8 / 88 /-7 /-7 / 88 / 3 /-87]$} \\
2.12 & 1.01 & {$[-83 / 87 / 7 /-3 / 87 /-3 /-3 /-3 / 7 / 87 /-3 / 87 /-3 / 87 / 7 / 87 /-83 /-3 /-83 /-3 /-3 / 7 /-83 / 87 / 87 / 7 / 87 /-3 /-3]$} \\
2.13 & 1.03 & {$[-87 /-2 / 88 / 88 / 8 / 88 /-7 /-7 / 8 /-2 /-7 / 8 /-2 /-2 / 88 / 88 /-87 / 88 /-87 /-7 / 8 / 88 /-87 / 88 /-2 /-2 / 88 / 8 /-7]$} \\
2.14 & 1.03 & {$[8 / 88 /-87 /-7 /-7 / 88 / 3 / 3 /-87 / 88 / 3 /-7 / 88 /-7 /-87 / 88 / 8 /-7 / 8 / 3 / 88 /-87 / 8 /-7 / 88 /-87 / 88 /-7 / 3]$} \\
2.15 & 1.01 & {$[-87 / 88 / 3 /-2 / 88 /-2 /-2 / 88 /-2 / 3 / 88 /-2 /-2 / 3 /-2 / 88 /-87 /-2 /-87 / 88 / 3 / 88 /-87 /-2 / 88 / 88 / 3 /-2 /-2]$} \\
\hline
\end{tabular}

Among the solutions listed in Table 7, the stacking sequence identified by the ID 2.01 has been selected to be manufactured and tested. The load-displacement curve for this 
Table 8: Mechanical properties of the optimum stacking sequences in terms of buckling load and membrane stiffness along $y$-axis; for each property the percentage difference between the optimum configuration and the reference one is indicated in parentheses.

\begin{tabular}{lll}
\hline ID & $\lambda_{N L}[\mathrm{~N}]$ & $A_{y y}[\mathrm{~N} / \mathrm{mm}]$ \\
\hline 2.01 & $50486(+3.5 \%)$ & $375219(+3.3 \%)$ \\
2.02 & $50551(+3.7 \%)$ & $374193(+3.0 \%)$ \\
2.03 & $50662(+3.9 \%)$ & $375105(+3.2 \%)$ \\
2.04 & $50581(+3.7 \%)$ & $374194(+3.0 \%)$ \\
2.05 & $50408(+3.4 \%)$ & $375303(+3.3 \%)$ \\
2.06 & $50202(+3.0 \%)$ & $375275(+3.3 \%)$ \\
2.07 & $50494(+3.6 \%)$ & $375219(+3.3 \%)$ \\
2.08 & $50600(+3.8 \%)$ & $375235(+3.3 \%)$ \\
2.09 & $50569(+3.7 \%)$ & $374482(+3.1 \%)$ \\
2.10 & $49598(+1.7 \%)$ & $375072(+3.2 \%)$ \\
2.11 & $49638(+1.8 \%)$ & $374591(+3.1 \%)$ \\
2.12 & $50072(+2.7 \%)$ & $371435(+2.2 \%)$ \\
2.13 & $49666(+1.9 \%)$ & $374627(+3.1 \%)$ \\
2.14 & $49694(+1.9 \%)$ & $374591(+3.1 \%)$ \\
2.15 & $50488(+3.5 \%)$ & $374233(+3.0 \%)$ \\
\hline
\end{tabular}

optimum solution (both experimental and numerical results) is given in Fig. 11.

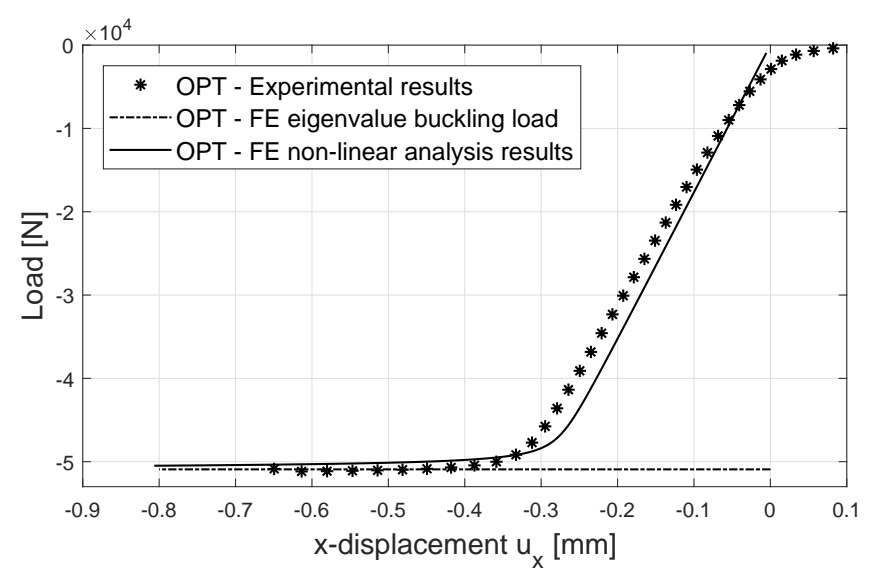

Figure 11: Experimental and numerical results comparison, optimum solution.

The polar diagram of stack 2.01 is shown in Fig. 12: only the first component of the normalised stiffness matrices of the laminate, i.e. $\mathbf{A}^{*}, \mathbf{B}^{*}$ and $\mathbf{D}^{*}$ are represented. The solid line refers to the membrane stiffness matrix, the dashed one to the bending stiffness matrix, while the dash-dotted one is linked to the membrane/bending coupling stiffness matrix. It can be noticed that the laminate is uncoupled as the dash-dotted curve disappears, homogeneous as the solid and dashed curves are coincident and orthotropic because there are two orthogonal axes of symmetry in the plane. In addition, for both laminates the main orthotropy axis is oriented at $\Phi_{1}^{A^{*}}=0^{\circ}$ according to the hypothesis of the firstlevel problem. Of course, the same considerations can be repeated also for the rest of the optimum solutions.

A summary of the results characterising the stack 2.01 is given in Table 9 . 


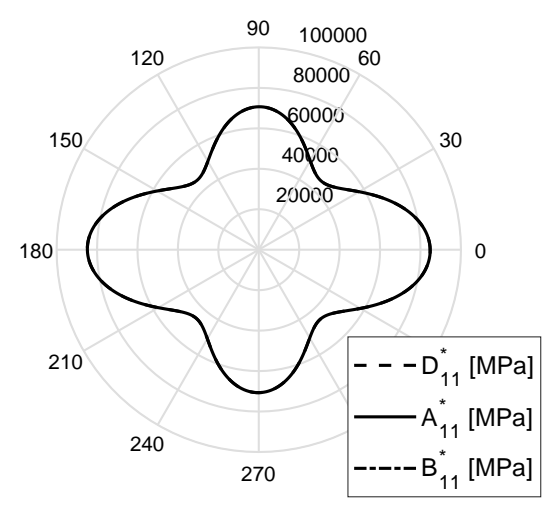

Figure 12: Polar diagram of stack 2.01.

Table 9: Numerical and experimental mechanical properties for the optimum solution 2.01; for each property the percentage difference between the optimum configuration and the reference one is indicated in parentheses.

\begin{tabular}{l|r}
\hline Property & Optimum solution \\
\hline$n_{\text {ply }}$ & $29(-9.4 \%)$ \\
$A_{y y}[\mathrm{~N} / \mathrm{mm}]$ & $375219(+3.3 \%)$ \\
$\lambda_{(\exp )}[\mathrm{N}]$ & $51189(+5.0 \%)$ \\
$\lambda_{L}[\mathrm{~N}]$ & $50922(+4.1 \%)$ \\
$\lambda_{N L}[\mathrm{~N}]$ & $50486(+3.6 \%)$ \\
\hline
\end{tabular}

Also for this case, experimental and numerical results are in excellent agreement with a percentage difference of $-1.4 \%$ (non-linear numerical buckling load over the experimental one), thus giving an experimental proof of the effectiveness of QT stacks and, by extension, of the MS2L optimisation process.

\section{Conclusions}

The design strategy presented in this paper is a numerical optimisation procedure characterised by several features that make it an innovative, effective and general method for the multi-scale design of composite structures. In the present work this strategy has been applied to the multi-scale optimisation of a composite multilayer plate.

The design process is not submitted to restrictions: any parameter characterising the structure (at each relevant scale) is an optimisation variable. This allows searching for a true global minimum, hard to be obtained otherwise. The multi-scale design problem has been split into two distinct but linked non-linear minimisation problems which are solved subsequently within the same numerical procedure. The first-level problem focuses on the macroscopic scale of the panel: the laminate is considered as an equivalent homogeneous anisotropic plate and its macroscopic mechanical response is described in terms of polar parameters. Furthermore, the overall plate thickness is also determined at this level. The second level of the procedure is devoted to find at least one optimum stack meeting the elastic requirements imposed to the laminate (quasi-homogeneity and orthotropy) as well as the optimum value of the laminate polar parameters resulting from the first step.

At the macroscopic scale, the mechanical properties of the multilayer plate are repre- 
sented by means of the polar formalism, a mathematical representation characterised by several advantages. The main features of the polar method are the possibility to represent in an explicit and straightforward way the elastic symmetries of the laminate stiffness matrices, the elastic and geometric bounds for the laminate polar parameters and to eliminate from the optimisation procedure redundant variables. In addition, the utilisation of the polar formalism leads the designer to easily formulate the second-level problem by taking into account in a correct and elegant way the requirements on the elastic symmetries of the structure, without making simplifying hypotheses on the nature of the stacking sequence.

As far as the optimisation calculations are concerned, they are carried out by a special genetic algorithm able to integrate both continuous and discrete-valued variables during the same calculation and to effectively handle the optimisation constraints by means of the very general ADP method. For the solution of the first-level problem, the GA has been interfaced with the FE commercial code ANSYS that invokes a linear eigenvalue buckling analysis in order to compute both the objective and the constraint functions of the problem.

The utilisation of an evolutionary strategy, together with the fact that the problem is stated in the most general sense, allows finding some non-conventional configurations that are more efficient than the standard ones. In fact, the considered example proves that, when standard rules for tailoring laminate stacks are abandoned and all the parameters characterising the laminate are included within the design process, a significant weight saving can be obtained: up to $9.4 \%$ (with respect to a reference canonical stack) with enhanced mechanical properties in terms of both first buckling load and membrane stiffness along $y$-axis (the percentage increment range from from $+1.7 \%$ to $+3.9 \%$ and from $+2.2 \%$ to $+3.3 \%$, respectively, depending on the considered optimum solution).

In addition, experimental buckling tests have been conducted on both reference and optimum solutions. Experimental and numerical results are in excellent agreement (maximum absolute percentage difference lower than 1.4\%) confirming, in this way, the high potential of non-conventional QT solutions and, by extension, that of the MS2L optimisation approach. These encouraging results unquestionably prove the effectiveness and the robustness of the MS2L optimisation approach proposed in this work and provide confidence for further research in this direction.

As far as the perspectives of this work are concerned, research is ongoing in order to develop a suitable global/local modelling approach for composite structures to be integrated into the MS2L optimisation strategy when applied to real-world engineering problems (as the multi-scale optimisation of a wing-box structure). A proper global/local modelling is of paramount importance when both global (e.g. mass, stiffness, etc.) and local (failure criteria, local buckling, etc.) design criteria must be included into the optimisation process. These activities will be developed in the framework of the project PARSIFAL (Prandtlplane ARchitecture for the Sustainable Improvement of Future AirpLanes) funded by the European Union.

\section{Acknowledgements}

This paper presents part of the activities carried out within the research project PARSIFAL (Prandtlplane ARchitecture for the Sustainable Improvement of Future AirpLanes), which has been funded by the European Union under the Horizon 2020 Research and Innovation Program (Grant Agreement n. 723149). 


\section{References}

[1] P. Trovalusci, Generalized Continua as Models for Classical and Advanced Materials, Vol. 42 of Advanced Structured Materials, Springer International Publishing, Switzerland, 2016, Ch. A Statistically-Based Homogenization Approach for Particle Random Composites as Micropolar Continua, pp. 425-441.

[2] E. Reccia, M. L. D. Bellis, P. Trovalusci, R. Masiani, Sensitivity to material contrast in homogenization of random particle composites as micropolar continua, Composites Part B: Engineering 136 (2018) $39-45$.

[3] L. Leonetti, F. Greco, P. Trovalusci, R. Luciano, R. Masiani, A multiscale damage analysis of periodic composites using a couple-stress/cauchy multidomain model: Application to masonry structures, Composites Part B: Engineering 141 (2018) 50 - 59.

[4] S. Adali, V. Verijenko, M. Walker, Optimal laminate configurations with symmetric lay-ups for maximum postbuckling stiffness, Composites Engineering 4 (11) (1994) $1119-1127$.

[5] S. Adali, A. Richter, V. Verijenko, E. Summers, Optimal design of hybrid laminates with discrete ply angles for maximum buckling load and minimum cost, Composite Structures 32 (1) (1995) 409 - 415, eighth International Conference on Composite Structures.

[6] S. Adali, M. Walker, V. Verijenko, Multiobjective optimization of laminated plates for maximum prebuckling, buckling and postbuckling strength using continuous and discrete ply angles, Composite Structures 35 (1) (1996) 117 - 130, stability of Composite Structures.

[7] S. Adali, F. Lene, G. Duvaut, V. Chiaruttini, Optimization of laminated composites subject to uncertain buckling loads, Composite Structures 62 (3) (2003) 261 - 269.

[8] R. T. HAFTKA, J. L. WALSH, Stacking-sequence optimization for buckling of laminated plates by integer programming, AIAA Journal 30 (3) (1992) 814-819.

[9] R. Le Riche, R. T. Haftka, Optimization of laminate stacking sequence for buckling load maximization by genetic algorithm, AIAA Journal 31 (5) (1993) 951-956.

[10] F. Aymerich, M. Serra, Optimization of laminate stacking sequence for maximum buckling load using the ant colony optimization (ACO) metaheuristic, Composites Part A: Applied Science and Manufacturing 39 (2) (2008) 262-272.

[11] F.-X. Irisarri, D. H. Bassir, N. Carrere, J.-F. Maire, Multiobjective stacking sequence optimization for laminated composite structures, Composites Science and Technology 69 (7-8) (2009) 983-990.

[12] U.S. Department of Defense, Military Handbook - MIL-HDBK-17-3F: Composite Materials Handbook, Volume 3 - Polymer Matrix Composites Materials Usage, Design and Analysis (2002).

[13] R. M. Jones, Mechanics of composite materials, McGraw-Hill, 1975.

[14] S. W. Tsai, N. J. Pagano, Invariant properties of composite materials., Tech. rep., AIR FORCE MATERIALS LAB WRIGHT-PATTERSON AFB OHIO (1968). 
[15] S. W. Tsai, T. Hahn, Introduction to composite materials, Technomic, 1980.

[16] C. G. Diaconu, M. Sato, H. Sekine, Feasible Region in General Design Space of Lamination Parameters for Laminated Composites, AIAA Journal 40 (3) (2002) 559-565.

[17] C. Diaconu, M. Sato, H. Sekine, Layup optimization of symmetrically laminated thick plates for fundamental frequencies using lamination parameters, Structural and Multidisciplinary Optimization 24 (4) (2002) 302-311.

[18] B. Liu, R. T. Hatfka, P. Trompette, Maximization of buckling loads of composite panels using flexural lamination parameters, Structural and Multidisciplinary Optimization 26 (2004) 28-36.

[19] M. Bloomfield, J. Herencia, P. Weaver, Optimisation of anisotropic composite plates incorporating non-conventional ply orientations, in: 49th AIAA/ASME/ASCE/AHS/ASC Structures, Structural Dynamics, and Materials Conference, 16th AIAA/ASME/AHS Adaptive Structures Conference, 10th AIAA Non-Deterministic Approaches Conference, 9th AIAA Gossamer Spacecraft Forum, 4th AIAA Multidisciplinary Design Optimization Specialists Conference, Schaumburg, IL, American Institute of Aeronautics and Astronautics, 2008, p. 1918.

[20] S. Liu, Y. Hou, X. Sun, Y. Zhang, A two-step optimization scheme for maximum stiffness design of laminated plates based on lamination parameters, Composite Structures 94 (12) (2012) 3529-3537.

[21] M. Montemurro, An extension of the polar method to the first-order shear deformation theory of laminates, Composite Structures 127 (2015) 328-339.

[22] S. Nagendra, D. Jestin, Z. Gürdal, R. Hatfka, L. Watson, Improved genetic algorithm for the design of stiffened composite panels, Computers and Structures 58 (3) (1996) $543-555$.

[23] R. Le Riche, R. T. Hatfka, Improved genetic algorithm for minimum thickness composite laminate design, Composites Engineering 5 (2) (1995) 143-161.

[24] A. Todoroki, R. T. Hatfka, Stacking sequence optimization by a genetic algorithm with a new recessive gene like repair strategy, Composites Part B 29 (1998) 277-285.

[25] G. Verchery, Mechanical Behavior of Anisotropic Solids / Comportment Mécanique des Solides Anisotropes, Springer Netherlands, 1982, Ch. Les invariants des tenseurs d'ordre 4 du type de l'élasticité, pp. 93-104, DOI: 10.1007/978-94-009-6827-1_7.

[26] A. Catapano, M. Montemurro, A multi-scale approach for the optimum design of sandwich plates with honeycomb core. Part I: homogenisation of core properties, Composite Structures 118 (2014) 664-676.

[27] A. Catapano, M. Montemurro, A multi-scale approach for the optimum design of sandwich plates with honeycomb core. Part II: the optimisation strategy, Composite Structures 118 (2014) 677-690.

[28] M. Montemurro, A. Catapano, D. Doroszewski, A multi-scale approach for the simultaneous shape and material optimisation of sandwich panels with cellular core, Composites Part B 91 (2016) 458-472. 
[29] M. Montemurro, Corrigendum to "an extension of the polar method to the first-order shear deformation theory of laminates" [compos. struct. 127 (2015) 328-339], Composite Structures 131 (2015) 1143-1144.

[30] M. Montemurro, The polar analysis of the third-order shear deformation theory of laminates, Composite Structures 131 (2015) 775-789.

[31] M. Montemurro, Optimal design of advanced engineering modular systems through a new genetic approach, Ph.D. thesis, UPMC, Paris VI, France, http://tel.archivesouvertes.fr/tel-00955533 (2012).

[32] M. Montemurro, A contribution to the development of design strategies for the optimisation of lightweight structures, Université de Bordeaux, France, 2018, HDR Thesis.

[33] M. Montemurro, A. Vincenti, P. Vannucci, Design of elastic properties of laminates with minimum number of plies, Mechanics of Composite Materials 48 (2012) 369-390.

[34] M. Montemurro, Y. Koutsawa, S. Belouettar, A. Vincenti, P. Vannucci, Design of damping properties of hybrid laminates through a global optimisation strategy, Composite Structures 94 (2012) 3309-3320.

[35] M. Montemurro, A. Vincenti, Y. Koutsawa, P. Vannucci, A two-level procedure for the global optimization of the damping behavior of composite laminated plates with elastomer patches, Journal of Vibration and Control 21 (9) (2015) 1778-1800.

[36] M. Montemurro, A. Catapano, Variational analysis and aerospace engineering: mathematical challenges for the aerospace of the future, 1st Edition, Vol. 116 of Springer Optimization and Its Applications, Springer International Publishing, 2016, Ch. A new paradigm for the optimum design of variable angle tow laminates, pp. 375-400, DOI: $10.1007 / 978-3-319-45680-5$.

[37] M. Montemurro, A. Catapano, On the effective integration of manufacturability constraints within the multi-scale methodology for designing variable angle-tow laminates, Composite Structures 161 (2017) 145-159.

[38] M. Montemurro, A. Catapano, A general B-Spline surfaces theoretical framework for optimisation of variable angle-tow laminates, Composite Structures 209 (2019) 561578.

[39] ASTM, West Conshohocken, Pa, ASTM, D3518/D3518M-94(07) Standard test method for in-plane shear response of polymer matrix composite materials by tensile test of a $\pm 45^{\circ}$ laminate, URL: www.astm.org (2007).

[40] M. I. Izzi, Multi-scale analysis, design and optimisation of multilayer plates, Master's thesis, Università di Pisa (2016).

[41] J. N. Reddy, Mechanics of composite laminated plates and shells: theory and analysis, Boca Raton, FL: CRC Press, 2003.

[42] P. Vannucci, Plane anisotropy by the polar method, Meccanica 40 (2005) 437-454.

[43] P. Vannucci, A note on the elastic and geometric bounds for composite laminates, Journal of Elasticity 112 (2013) 199-215. 
[44] M. Montemurro, A. Vincenti, P. Vannucci, The automatic dynamic penalisation method (ADP) for handling constraints with genetic algorithms, Computer Methods in Applied Mechanics and Engineering 256 (2013) 70-87.

[45] M. Montemurro, H. Nasser, Y. Koutsawa, S. Belouettar, A. Vincenti, P. Vannucci, Identification of electromechanical properties of piezoelectric structures through evolutionary optimisation techniques, International Journal of Solids and Structures 49 (13) (2012) 1884-1892.

[46] G. Costa, M. Montemurro, J. Pailhès, A General Hybrid Optimization Strategy for Curve Fitting in the Non-Uniform Rational Basis Spline Framework, Journal of Optimization Theory and Applications 176 (1) (2018) 225-251.

[47] P. Vannucci, G. Verchery, A special class of uncoupled and quasi-homogeneous laminates, Composites Science and Technology 61 (2001) 1465-1473.

[48] ANSYS, Inc., 275 Technology Drive, Canonsburg, PA 15317, ANSYS Mechanical APDL Modeling and Meshing Guide (2012). 